Bull. Austral. Math. Soc.

Vol. 54 (1996) [267-274]

\title{
THE SELMER GROUPS AND THE AMBIGUOUS IDEAL CLASS GROUPS OF CUBIC FIELDS
}

\author{
YEN-MEI J. CHEN
}

In this paper, we study a family of elliptic curves with CM by $\mathbb{Q}(\sqrt{-3})$ which also admits a $\mathbb{Q}$-rational isogeny of degree 3. We find a relation between the Selmer groups of the elliptic curves and the ambiguous ideal class groups of certain cubic fields. We also find some bounds for the dimension of the 3-Selmer group over $\mathbb{Q}$, whose upper bound is also an upper bound of the rank of the elliptic curve.

\section{INTRODUCTION}

Let $D$ be a cube-free integer. We consider the elliptic curve

$$
E / \mathbb{Q}: y^{2}=x^{3}+D^{2},
$$

which has $j$-invariant 0 and has complex multiplication $\pi=\sqrt{-3}$. More precisely, $\pi$ is the endomorphism

$$
\begin{aligned}
\pi: E / \mathbb{Q} & \rightarrow E / \mathbb{Q} \\
(x, y) & \mapsto\left(-\frac{x^{3}+4 D^{2}}{3 x^{2}},-\frac{y\left(x^{3}-8 D^{2}\right)}{3 \sqrt{-3} x^{3}}\right) .
\end{aligned}
$$

We set the following notation.

$$
\begin{aligned}
S_{1} & =\{p \text { prime }: p \mid D \text { and } p \equiv 1 \bmod 3\} \\
S_{2} & =\{p \text { odd prime }: p \mid D \text { and } p \equiv 2 \bmod 3\} \\
l_{1} & =\left|S_{1}\right| \\
l_{2} & =\left|S_{2}\right| \\
k & =\mathbb{Q}(\omega), \omega=\frac{1+\sqrt{-3}}{2} \\
K & =k(\sqrt[3]{2 D}) \\
U_{k} & =\text { the group of units of } k \\
U_{K} & =\text { the group of units of } K \\
C_{K} & =\text { the 3-class group of } K \\
C_{K}^{(r)} & =\left\{a \in C_{K}: a^{\tau}=a\right\},
\end{aligned}
$$

Received 1st November, 1995.

Copyright Clearance Centre, Inc. Serial-fee code: 0004-9729/96 \$A2.00+0.00. 
where $\tau$ is a generator of the Galois group of the field extension $K / k$ and $C_{K}^{(\tau)}$ is called the ambiguous ideal class group of $K / k$. We first define a map

$$
\Psi: S^{(\pi)}(E / k) \longrightarrow C_{K}^{(\tau)}
$$

then we can obtain an upper bound of the rank of the Selmer group $S^{(3)}(E / \mathbb{Q})$ by using the theorem of Gerth [3] which gives an explicit computation of the rank of the group $C_{K}^{(\tau)}$. On the other hand, we can obtain a lower bound by using the duality theorem of Cassels [1]. More precisely, we can obtain the following inequalities:

$$
l_{2}+\varepsilon_{2}-3 \leqslant \operatorname{dim}_{\mathbb{T}_{3}} S^{(3)}(E / \mathbb{Q}) \leqslant 2 l_{1}+l_{2}-\varepsilon_{1}+1
$$

where $\varepsilon_{1}$ and $\varepsilon_{2}$, both depending on $D$, are integers 0,1 , or 2 . For the family of curves $E / \mathbb{Q}: y^{2}=x^{3}+D^{3}$, Frey [2] showed that the rank of the Selmer group of a 3-isogeny is closely related to the class number of the quadratic field $\mathbb{Q}(\sqrt{D})$. Also Jan Nekevár [4] proved some analogous results for the elliptic curve given by $D y^{2}=4 x^{3}-27$ which is isomorphic to the curve given by $y^{2}=x^{3}-432 D^{3}$. Our result gives explicit bounds for the dimension of $S^{(3)}(E / k)$ and implies that the dimension can be arbitrarily large.

\section{The Selmer Group $S^{(\pi)}(E / k)$}

Definition: Let $F$ be a number field and let $\phi: E / F \rightarrow E^{\prime} / F$ be an isogeny defined over $F$. Then the $\phi$-Selmer group of $E / F$ is the subgroup of $H^{1}\left(G_{\bar{F} / F}, E[\phi]\right)$ defined by

$$
S^{(\phi)}(E / F) \stackrel{\text { def }}{=} \operatorname{ker}\left\{H^{1}\left(G_{\bar{F} / F}, E[\phi]\right) \rightarrow \prod_{v \in M_{F}} H^{1}\left(G_{\bar{F}_{v} / F_{v}}, E\right)\right\}
$$

Observe that the map $\pi: E / \mathbb{Q} \longrightarrow E / \mathbb{Q}$ given as above is defined over $k$ but not over $\mathbb{Q}$ and that $E[\pi]$ is isomorphic to $\mu_{3}$ as a $\mathrm{Gal}(\bar{k} / k)$-module, and thus we have

$$
H^{1}\left(G_{\bar{k} / k}, E[\pi]\right) \cong k^{*} / k^{* 3}
$$

Given an element $d \in k^{*}$, it corresponds to the homogeneous space of $E$ which can be given by

$$
C_{d}: d x^{3}+d^{2} y^{3}=2 D z^{3}
$$

Then such $d$ will be an element of the Selmer group $S^{(\pi)}(E / k)$ provided that $C_{d}$ admits a $k_{v}$-rational point for all $v \in M_{k}$. For any such $d$, since $2 D$ is a perfect cube in $K$ 
the principal divisor $(d)$ must be a cube of some divisor in $K$, say $(d)=\mathfrak{a}^{3}$. It is clear that $\mathfrak{a}^{\tau}=\mathfrak{a}$, so $\mathfrak{a} \in C_{K}^{(\tau)}$. Thus we can define a homomorphism

$$
\Psi: S^{(\pi)}(E / k) \longrightarrow C_{K}^{(\tau)}
$$

by $\Psi(d)=\mathrm{a}$. Then it is clear that $\operatorname{ker} \Psi=U_{K} \cdot K^{* 3} \cap k^{*} / k^{* 3}$. Note that $\Psi$ induces two maps

$$
\begin{aligned}
& \Psi^{+}: S^{(\pi)}(E / k)^{+} \longrightarrow C_{K}^{(\tau)^{+}}, \\
& \Psi^{-}: S^{(\pi)}(E / k)^{-} \longrightarrow C_{K}^{(\tau)^{-}}
\end{aligned}
$$

where + and - refer to the action of $\mathrm{Gal}(k / \mathbb{Q})$. Observe that all of the groups mentioned above are $\mathbb{F}_{3}$-vector spaces.

LEMma 1.1 . (Gerth)

(a) $\operatorname{dim}_{\mathbb{F}_{3}} C_{K}^{(\tau)}=2 l_{1}+l_{2}-\varepsilon_{1}$;

(b) $\operatorname{dim}_{\mathbb{F}_{3}} C_{K}^{(\tau)^{+}}=l_{1}$;

(c) $\operatorname{dim}_{\mathbb{F}_{3}} C_{K}^{(\tau)^{-}}=l_{1}+l_{2}-\varepsilon_{1}$;

where $\varepsilon_{1}$ (depending on $D$ ) is 0,1 or 2 .

Proof: See [3].

Lemma 1.2 .

(a) $\operatorname{dim}_{\mathbb{R}_{3}} S^{(\pi)}(E / k) \leqslant \operatorname{dim}_{\mathbb{Y}_{3}} C_{K}^{(\tau)}+2$.

(b) $\operatorname{dim}_{\mathbb{Y}_{3}} S^{(\pi)}(E / k)^{+} \leqslant \operatorname{dim}_{\mathbb{Z}_{3}} C_{K}^{(\tau)+}+1$.

(c) $\operatorname{dim}_{\mathbb{F}_{3}} S^{(\pi)}(E / k)^{-} \leqslant \operatorname{dim}_{\mathbb{F}_{3}} C_{K}^{(\tau)^{-}}+1$.

Proof: (a) We already see that $\Psi$ is a homomorphism from $S^{(\pi)}(E / k)$ to $C_{K}^{(\tau)}$ with $\operatorname{ker} \Psi=U_{K} \cdot K^{* 3} \cap k^{*} / k^{* 3}$. Since $U_{K} \cdot K^{* 3} \cap k^{*}=U_{k} \cdot K^{* 3} \cap k^{*}$, we have $U_{K} \cdot K^{* 3} \cap k^{*} / k^{* 3}=\left\{1,2 D, 4 D^{2}\right\} \cdot U_{k} \cdot k^{* 3} / k^{* 3}$. The Dirichlet Unit Theorem implies that $U_{k}=\mu_{6}$. Hence $\operatorname{dim}_{\mathbb{F}_{3}} \operatorname{ker} \Psi=2$ and and thus we have

$$
\operatorname{dim}_{\mathbb{F}_{3}} S^{(\pi)}(E / k) \leqslant \operatorname{dim}_{\mathbb{P}_{3}} C_{K}^{(\tau)}+2 .
$$

(b) Observe that $\operatorname{ker} \Psi^{+}$is generated by $\{2 D\}$, and thus $\operatorname{dim}_{\mathbb{T}_{3}} \operatorname{ker} \Psi^{+}=1$. Therefore (b) holds.

(c) Similar to (b) except that $\operatorname{ker} \Psi^{-}$is generated by $\{\omega\}$.

Proposition 1.3.

(a) $\operatorname{dim}_{\mathbb{P}_{3}} S^{(\pi)}(E / k) \leqslant l_{1}+l_{2}-\varepsilon_{1}+2$.

(b) $\operatorname{dim}_{T_{3}} S^{(\pi)}(E / k)^{+} \leqslant l_{1}+1$.

(c) $\operatorname{dim}_{\Gamma_{3}} S^{(\pi)}(E / k)^{-} \leqslant l_{1}+l_{2}-\varepsilon_{1}+1$.

Proof: Follows immediately from Lemma 1.1 and Lemma 1.2. 


\section{The Selmer Group $S^{(3)}(E / k)$}

Recall that $\pi^{2}=-3$, so we have the following exact sequence

$$
0 \longrightarrow E[\pi] \hookrightarrow E[3] \stackrel{\pi}{\longrightarrow} E[\pi] \longrightarrow 0 .
$$

Taking Galois cohomology as $G_{K / k}, G_{\bar{k} / k}$, and $G_{\bar{K} / K^{-m o d u l e s ~ r e s p e c t i v e l y, ~ w e ~ k n o w ~}}$ that each row of the following commutative diagram is exact except at the end. Since we can view $G_{\bar{K} / K}$ as a subgroup of $G_{\bar{k} / k}$, the Inf-Res sequence implies that each column is also exact.

$$
\begin{aligned}
& \begin{array}{rrrr}
0 & 0 & 0 \\
\downarrow & \downarrow & \downarrow
\end{array} \\
& 0 \rightarrow E[\pi] \rightarrow H^{1}\left(G_{K / k}, E[\pi]\right) \rightarrow H^{1}\left(G_{K / k}, E[3]\right) \stackrel{\tilde{\pi}}{\rightarrow} H^{1}\left(G_{K / k}, E[\pi]\right) \stackrel{?}{\rightarrow} 0 \\
& 0 \rightarrow E[\pi] \rightarrow H^{1}\left(G_{\bar{k} / k}, E[\pi]\right) \rightarrow H^{1}\left(G_{\bar{k} / k}, E[3]\right) \stackrel{\tilde{\pi}^{\prime}}{\rightarrow} H^{1}\left(G_{\bar{k} / k}, E[\pi]\right) \stackrel{?}{\rightarrow} 0 \\
& \downarrow \quad \downarrow \quad \downarrow \\
& 0 \rightarrow H^{1}\left(G_{\bar{K} / K}, E[\pi]\right)^{G_{K / k}} \rightarrow H^{1}\left(G_{\bar{K} / K}, E[3]\right)^{G_{K / k}} \stackrel{\underset{\pi}{\rightarrow}}{\rightarrow} H^{1}\left(G_{\bar{K} / K}, E[\pi]\right)^{G_{K / k}} \stackrel{?}{\rightarrow} 0 \\
& \downarrow \\
& 0 \rightarrow H^{2}\left(G_{K / k}, E[\pi]\right) \rightarrow H^{2}\left(G_{K / k}, E[\pi]\right) \rightarrow H^{2}\left(G_{K / k}, E[\pi]\right) \rightarrow \cdots \\
& \downarrow
\end{aligned}
$$

By routine computations, we have the following equalities:

$$
\begin{aligned}
& H^{1}\left(G_{K / k}, E[\pi]\right) \cong H^{2}\left(G_{K / k}, E[\pi]\right) \cong \mathbb{Z} / 3 \mathbb{Z}, \\
& H^{1}\left(G_{K / k}, E[3]\right) \cong H^{2}\left(G_{K / k}, E[3]\right) \cong \mathbb{Z} / 3 \mathbb{Z} .
\end{aligned}
$$

Then it is clear that the first row is exact. Note that $E[3]$ is isomorphic to $\mu_{3} \times \mu_{3}$ as a $\mathrm{Gal}(\bar{K} / K)$-module; thus we have

$$
H^{1}\left(G_{\bar{K} / K}, E[3]\right) \cong K^{*} / K^{* 3} \times K^{*} / K^{* 3} .
$$

The third row is equivalent to the following exact sequence, and therefore it is exact.

$$
\begin{aligned}
0 \longrightarrow K^{*} / K^{* 3} & \longrightarrow K^{*} / K^{* 3} \times K^{*} / K^{* 3} \\
a & \mapsto(a, 1) \quad(a, b) \mapsto b
\end{aligned}
$$

Combining all the observations above, we have the following lemma: 
LEMma 2.1. The $\mathbb{F}_{3}$-dimension of the cokernel of the map

$$
\tilde{\pi}: H^{1}\left(G_{\bar{k} / k}, E[3]\right) \rightarrow H^{1}\left(G_{\bar{k} / k}, E[\pi]\right)
$$

is less than or equal to 1 .

Proposition 2.2. The $\mathbb{F}_{3}$-dimension of the cokernel of the map

$$
\tilde{\pi}: S^{(3)}(E / k) \longrightarrow S^{(\pi)}(E / k)
$$

is less than or equal to 2 .

PROOF: Given arbitrary $a \in S^{(\pi)}(E / k)$ - in other words the corresponding homogeneous space is locally trivial everywhere - it is easy to check that at least one of $a, 2 D a, 4 D^{2} a$ is locally a cube everywhere except at $v, v \mid 3$. If $a$ is in the image of the map $\tilde{\pi}: H^{1}\left(G_{\bar{k} / k}, E[3]\right) \rightarrow H^{1}\left(G_{\bar{k} / k}, E[\pi]\right)$ and it is locally a cube at $v, v \mid 3$, then $(1, a) \in S^{(3)}(E / k)$ and $\tilde{\pi}((1, a))=a$. It is easy to see that given a finite set $T$ of indepedent elements in $k^{*} / k^{* 3}$ one can find another set $T^{\prime}$ such that $T$ and $T^{\prime}$ generate the same subgroup in $k^{*} / k^{* 3}$ and every element in $T^{\prime}$ is a cube at $v, v / 3$ with at most one exception. Therefore Lemma 2.1 implies that the $\mathbb{F}_{3}$-dimension of the cokernel of the map $S^{(3)}(E / k) \stackrel{\tilde{\pi}}{\rightarrow} S^{(\pi)}(E / k)$ is less than or equal to 2 .

Corollary 2.3. Assume that $\amalg(E / k)\left[3^{\infty}\right]$ is finite. Assume that either $D$ is not divisible by 3 or $D$ is divisible by 9 . Then the sequence

$$
0 \rightarrow E[\pi] \rightarrow S^{(\pi)}(E / k) \rightarrow S^{(3)}(E / k) \stackrel{\widetilde{\pi}}{\rightarrow} S^{(\pi)}(E / k) \rightarrow 0
$$

is exact.

Proof: It suffices to show that

$$
S^{(3)}(E / k) \stackrel{\tilde{\pi}}{\rightarrow} S^{(\pi)}(E / k) \rightarrow 0
$$

is exact. Given arbitrary $a \in S^{(\pi)}(E / k)$, the second hypothesis implies that $a$ is locally a cube at $v, v \mid 3$. Thus $(1, a) \in S^{(3)}(E / k)$ and $\tilde{\pi}((1, a))=a$. Again according to Lemma 2.1, we know that $\mathbb{F}_{3}$-dimension of the cokernel of the map $S^{(3)}(E / k) \stackrel{\widetilde{\pi}}{\rightarrow}$ $S^{(\pi)}(E / k)$ is less than or equal to 1 . Now the first hypothesis implies that $S^{(3)}(E / k) \stackrel{\tilde{\pi}}{\rightarrow}$ $S^{(\pi)}(E / k)$ is surjective if and only if $\operatorname{dim}_{I_{3}} S^{(3)}(E / k)$ is odd. Therefore we need the following lemma to complete the proof.

LEMMA . $\operatorname{dim}_{\mathbb{F}_{3}} S^{(3)}(E / k)$ is odd.

Proof: $1^{0}$ There is an exact sequence

$$
0 \longrightarrow E(k) / 3(E(k)) \longrightarrow S^{(3)}(E / k) \longrightarrow \amalg(E / k)[3] \longrightarrow 0
$$


which implies $\operatorname{dim}_{\mathbb{F}_{3}} S^{(3)}(E / k)$ and $\operatorname{dim}_{\mathbb{T}_{3}} E(k) / 3(E(k))$ have the same parity, thus it suffices to show that $\operatorname{dim}_{\mathbb{F}_{3}} E(k) / 3(E(k))$ is odd.

$2^{0}$ Consider the following sequence:

$$
\begin{gathered}
0 \longrightarrow E(\mathbb{Q}) \stackrel{\alpha}{\longrightarrow} E^{\prime}(k) \stackrel{\beta}{\longrightarrow} E^{\prime}(\mathbb{Q}) \longrightarrow 0 \\
(x, y) \mapsto P=(-3 x,-3 \sqrt{-3 y}) \mapsto P+P^{\sigma}
\end{gathered}
$$

where $E^{\prime}$ is given by $E^{\prime} / \mathbb{Q}: y^{2}=x^{3}-27 D^{2}$ and is isogeneous to the original curve $E$. We claim that the sequence is exact. It is clear that $\alpha$ is injective and that $\operatorname{ker} \beta=\operatorname{im} \alpha$. We show that $\beta$ is surjective. Given any point $Q=(x, y) \in E^{\prime}(\mathbb{Q})$, then $P=(x \omega,-y)$ and $P^{\sigma}=\left(x \omega^{2},-y\right)$ are both $k$-rational points. By an easy computation, we have $Q=P+P^{\sigma}=\beta(P)$, and so $\beta$ is surjective.

Since the group $E^{\prime}(\mathbb{Q})$ is torsion-free and finitely generated, it is a projective $\mathbb{Z}$ module, and thus the above sequence splits. By taking tensor products with the group $\mathbb{Z} / 3 \mathbb{Z}$, we obtain another exact sequence

$$
0 \rightarrow E(\mathbb{Q}) / 3 E(\mathbb{Q}) \rightarrow E^{\prime}(k) / 3 E^{\prime}(k) \rightarrow E^{\prime}(\mathbb{Q}) / 3 E^{\prime}(\mathbb{Q}) \rightarrow 0 .
$$

Therefore we have

$$
\begin{aligned}
\operatorname{dim}_{\mathbb{F}_{3}} E(k) / 3(E(k)) & =\operatorname{dim}_{\mathbb{F}_{3}} E(\mathbb{Q}) / 3 E(\mathbb{Q})+\operatorname{dim}_{\mathbb{F}_{3}} E^{\prime}(\mathbb{Q}) / 3 E^{\prime}(\mathbb{Q}) \\
& =2 \operatorname{dim}_{\mathbb{F}_{3}} E^{\prime}(\mathbb{Q}) / 3 E^{\prime}(\mathbb{Q})+1 .
\end{aligned}
$$

(Since $E_{\text {tors }}(\mathbb{Q}) \cong \mathbb{Z} / 3 \mathbb{Z}$ and $\operatorname{rank}(E(\mathbb{Q}))=\operatorname{rank}\left(E^{\prime}(\mathbb{Q})\right)$.)

\section{Bounds for The Dimension of The Selmer Group $S^{(3)}(E / \mathbb{Q})$}

Now we turn to consider the 3 -isogeny

$$
\begin{aligned}
\lambda: E / \mathbb{Q} & \longrightarrow E^{\prime} / \mathbb{Q} \\
(x, y) & \mapsto\left(\frac{x^{3}+4 D^{2}}{x^{2}}, \frac{y\left(x^{3}-8 D^{2}\right)}{x^{3}}\right)
\end{aligned}
$$

and its dual

$$
\begin{aligned}
\hat{\lambda}: E^{\prime} / \mathbb{Q} & \rightarrow E / \mathbb{Q} \\
(x, y) & \mapsto\left(\frac{x^{3}+4 D^{2}}{81 x^{2}}, \frac{y\left(x^{3}-216 D^{2}\right)}{729 x^{3}}\right) .
\end{aligned}
$$

Then we can identify

$$
\begin{aligned}
& S^{(\lambda)}(E / \mathbb{Q})=S^{(\pi)}(E / k)^{+}, S^{(\hat{\lambda})}\left(E^{\prime} / \mathbb{Q}\right)=S^{(\pi)}(E / k)^{-}, \\
& S^{(3)}(E / \mathbb{Q})=S^{(3)}(E / k)^{+} .
\end{aligned}
$$

Denote the dimensions of $S^{(\lambda)}(E / \mathbb{Q}), S^{(\hat{\lambda})}\left(E^{\prime} / \mathbb{Q}\right), S^{(3)}(E / \mathbb{Q})$ by $s, s^{\prime}, t$ respectively. Now we state the duality theorem of Cassels, which will be used latter. 
THEOREM. (Cassels [1])

$$
\frac{\left|S^{(\lambda)}(E / \mathbb{Q})\right|}{\left|S^{(\hat{\lambda})}\left(E^{\prime} / \mathbb{Q}\right)\right|}=\frac{\left|E_{\text {tors }}(\mathbb{Q})\right|}{\left|E_{\text {tors }}^{\prime}(\mathbb{Q})\right|} \cdot \prod_{p} \frac{c_{p}^{\prime}}{c_{p}} \cdot \frac{\int_{E^{\prime}(\mathbb{R})}\left|\omega_{\min }^{\prime}\right|_{\infty}}{\int_{E(\mathbb{R})}\left|\omega_{\min }\right|_{\infty}} .
$$

Lemma 3.1. $s-s^{\prime}=-l_{2}-\varepsilon_{2}$ where $\varepsilon_{2}$ depending on $D$ is $-2,-1,0$ or 1 .

PROOF: $1^{0}$ By elementary calculation,

$$
\frac{\int_{E^{\prime}(\mathbb{R})}\left|\omega_{\min }^{\prime}\right|_{\infty}}{\int_{E(\mathbb{B})}\left|\omega_{\min }\right|_{\infty}}=\frac{1}{3}
$$

$2^{0}$ By using the Tate's algorithm [5], we can obtain the following equalities :

$$
\begin{gathered}
\text { if } p \nmid 6 D, c_{p}=c_{p}^{\prime}=1 ; \quad \text { if } p \mid D, p \neq 2,3, \quad \frac{c_{p}}{c_{p}^{\prime}}= \begin{cases}3 & \text { if } p \equiv 2 \bmod 3, \\
1 & \text { if } p \equiv 1 \bmod 3 ;\end{cases} \\
\frac{c_{2}}{c_{2}^{\prime}}=\left\{\begin{array}{lll}
3 & \text { if D is odd }, \\
1 & \text { if D is even ; }
\end{array} \quad \frac{c_{3}}{c_{3}^{\prime}}= \begin{cases}3 & \text { if } 3 \mid D, \\
1 & \text { if } D \equiv 1,2,4,8 \bmod 9, \\
\frac{1}{3} & \text { if } D \equiv 5,7 \bmod 9 .\end{cases} \right.
\end{gathered}
$$

By combining all the above equalities, Lemma 3.1 will follow.

Finally, we obtain an upper bound and a lower bound for the dimension of the Selmer group $S^{(3)}(E / \mathbb{Q})$.

Proposition 3.2. $l_{2}+\varepsilon_{2}-3 \leqslant t \leqslant 2 l_{1}+l_{2}-\varepsilon_{1}+1$

Proof: $1^{0}$ According to Proposition 2.2 we already know that the sequence

$$
0 \rightarrow E[\pi] \rightarrow S^{(\pi)}(E / k) \rightarrow S^{(3)}(E / k) \stackrel{\tilde{\pi}}{\rightarrow} S^{(\pi)}(E / k)
$$

is exact and $\operatorname{dim}$ coker $\widetilde{\pi} \leqslant 2$. By considering the Galois group $\mathrm{Gal}(k / \mathbb{Q})$ acting on each group, we obtain another exact sequence

$$
0 \rightarrow E[\lambda] \rightarrow S^{(\lambda)}(E / \mathbb{Q}) \rightarrow S^{(3)}(E / \mathbb{Q}) \stackrel{\widetilde{\lambda}}{\rightarrow} S^{(\widetilde{\lambda})}\left(E^{\prime} / \mathbb{Q}\right)
$$

with $\operatorname{dim}$ coker $\tilde{\lambda} \leqslant 2$. Thus

$$
s+s^{\prime}-3 \leqslant t \leqslant s+s^{\prime}-1 .
$$

$2^{0}$ By combining Lemma 3.1 and Proposition 1.3, we have

$$
l_{2}+\varepsilon_{2}-3 \leqslant t \leqslant 2 l_{1}+l_{2}+\varepsilon_{1}+1 .
$$

Thus the proposition holds. 


\section{REFERENCES}

[1] J.W.S. Cassels, 'Arithmetic on Curves of Genus 1', J. Reine Angew. Math. 217 (1965), 180-199.

[2] G. Frey, 'Die Klassgruppe Quadratischer und Kubischer Zahlkörper und die Selmer Gruppen Gewisser Elliptische Kurven', Manuscripta Math. 16 (1975), 333-362.

[3] F. Gerth, 'On 3-class groups of pure cubic fields', J. Reine Angew. Math. 278/279 (1975), 52-62.

[4] J. Nekeváŕ, 'Class number of quadratic fields and Shimura's correspondence', Math. Ann. 287 (1990), 577-594.

[5] J. Tate, 'Algorithm for determining the type of a singular fiber in an elliptic pencil', in Lecture Notes Mathematics 476 (Springer-Verlag, Berlin, Heidelberg, New York, 1975), pp. 33-52.

\footnotetext{
Department of Mathematics

Tamkang University

Tamshui, Taipei 25137

Taiwan

Republic of China

e-mail: ymjchen@mail.tku.edu.tw
} 